\title{
Onlinemärkte für Musiksamples und die Fixierung flüchtiger Waren
}

\author{
Konstantin Hondros"
}

\section{Samples als flüchtige Waren}

Digitalisierung beeinflusst wirtschaftliche und künstlerische Praktiken auf vielfältige Weise. Während aus wirtschaftlicher Sicht die Schaffung neuer Märkte Bedeutung hat ${ }^{1}$, zeichnen sich künstlerische Praktiken im Digitalen hinsichtlich des erweiterten Möglichkeitsraumes aus, Neues aus Vorbestehendem zu schaffen ${ }^{2}$ - ein trügerischer perfect match, wie ich am Beispiel der Onlinemärkte für Musiksamples bespreche. Vor dem Hintergrund der Vereinheitlichung digitaler Märkte durch die Richtlinienreform der EU über das Urheberrecht im digitalen Binnenmarkt untersuche ich, ob und inwiefern es sich bei Musiksamples um Waren handelt und wie sie online dazu gemacht werden. Samples verstehe ich dabei als Musikfragmente, die hergestellt worden sind, um in Musikproduktionen verwendet zu werden.

In meiner Untersuchung nehme ich einen heuristischen Blickwinkel auf Samples ein und untersuche sie als potentiell flüchtige Waren. Mit dem Konzept der Flüchtigkeit greife ich auf eine Begrifflichkeit zurück, die bisher weder in der Analyse von Waren, noch in der Literatur zu Sampling eine ausgewiesene Rolle gespielt hat. Ich verspreche mir davon, eine rechtliche mit einer ästhetischen Dimension von Samples zu verbinden, die beide für Musiksamples und ebenso für weitere Waren in einer an Remix ausgerichteten, digitalen Ökonomie von Bedeutung sind. Für Onlinemärkte möchte ich mit meinem Beitrag zeigen, dass Musiksamples auf diesen zumeist fixiert werden und damit als entflüchtigt zu betrachten sind. Der per-

* Konstantin Hondros (M.A, Mag.) ist Soziologe und Theaterwissenschaftler und arbeitet als wissenschaftlicher Mitarbeiter an der Universität Duisburg-Essen. Er forscht zu kreativen Prozessen, Praktiken musikalischer Ähnlichkeit und regulatorischen Unsicherheiten mit Urheberrechtsbezug.

1 Goodman Berkeley Technology Law Journal 2004, 1389.

2 Knobel und Lankshear Journal of adolescent \& adult literacy 2008, 22. 
fect match zwischen neuen Märkten und erweitertem Möglichkeitsraum erfüllt sich häufig nicht.

Mit Flüchtigkeit orientiere ich mich zunächst allgemein an der Analyse der Moderne von Bauman ${ }^{3}$. Bauman beschreibt Flüchtigkeit als eine gesellschaftliche Verfasstheit, in der überkommene Ordnungen durch Fragmentierungen abgelöst werden. Wo zuvor voneinander räumlich und zeitlich Abgegrenztes, Bauman spricht von „solids“4, Handlungssicherheit versprochen hat, führt Flüchtigkeit zu Übergängen zwischen vormals Getrenntem, was von weitreichenden Unsicherheiten begleitet ist. Baumans Verständnis von Flüchtigkeit ist für Musiksamples insofern anschlussfähig, als es auf ihre Herstellungspraktik verweist, die im Auswählen und Kuratieren von Musikfragmenten liegt, und mit Unsicherheiten verbunden ist ${ }^{5}$. Das Mixen von Fragmenten durch Remix stellt eine mögliche Überwindung ästhetischer und ebenso rechtlicher Ordnungen dar, durch die sich zuvor klar gezogene Trennungen zwischen musikalischen Werken, wie sie das Urheberrecht vornimmt, als räumlich und zeitlich aufgebrochen erweisen.

Ausgehend von dieser zeitdiagnostischen Einbettung nehme ich mit Flüchtigkeit eine vorübergehende Zeitlichkeit in den Blick, die ich für die ästhetische Herstellung von Musiksamples konstatiere. Dabei beziehe ich mich auf die Untersuchung der Performativität theatraler Ästhetik. Für diese ist eine „Flüchtigkeit von Aufführungen“ ausgearbeitet worden, mit der die Bedeutung des Ephemeren in der Kunstproduktion unterstrichen wird ${ }^{6}$. Flüchtigkeit ist aus dieser Perspektive eine eigene ästhetische Qualität, der durch Fixierung, bspw. der digitalen Aufzeichnung einer Aufführung am Theater, nur teilweise beizukommen ist ${ }^{7}$. Auf diese Flüchtigkeit in der Kunstproduktion möchte ich hinaus, wenn ich Musiksamples als Waren verstehe, die in performativen Akten der Herstellung, Auswahl und damit des Kuratierens von Musikfragmenten entstehen.

Im Unterschied zu anderen Waren, aus kreativpraktischer Sicht etwa Pinsel und Farben, sind Samples flüchtig, weil sie - zumindest idealtypisch - erst im Zusammenhang ihrer ästhetischen Nutzung hergestellt werden und davor ein Teil eines anderen Musikstückes, und nicht primär als Musiksample angelegt waren. Literatur zu Musiksamples weist auf die „einzig-

3 Bauman, Liquid modernity, 2.

4 Ibid.

5 Hondros, Organized Creativity Working Paper Series, 20/1 2020, 1.

6 Fischer-Lichte, Ästhetik des Performativen, 127.

7 Zanetti und Heine, Transaktualität, 9. 
artige Momentaufnahme“ ${ }^{8}$ konservierter Klänge, das auf einem Datenträger fixierte „unique sound event "9 hin. Im Unterschied dazu fokussiere ich mit meinen Überlegungen zur Flüchtigkeit den Prozess der Dekontextualisierung, in dem ein Musiksample hergestellt und ausgewählt wird. Auf der einen Seite erschwert die Flüchtigkeit von Samples es, einen Markt um diese Waren zu entwickeln, der den Praktiken von Musikschaffenden entspricht. Auf der anderen Seite sind es aber gerade diese Praktiken, die kreative Nutzungen von Samples auszeichnen ${ }^{10}$. Bei diesen Nutzungen von Samples handelt es sich um Sampling, also die kreativen Praktiken, die mit Musiksamples in Verbindung stehen. ${ }^{11}$ Die Herstellung von Musiksamples kann, muss aber nicht mit Sampling als musikalischer Praktik einhergehen.

Im Mittelpunkt meiner Betrachtung stehen Onlinemärkte für $\mathrm{Mu}-$ siksamples, auf denen ich eine Entflüchtigung der potentiell flüchtigen $\mathrm{Mu}$ siksamples bemerke. Dieses Argument führe ich in meinem Beitrag aus, indem ich zunächst wirtschaftssoziologische Überlegungen zu Waren und Märkten vorstelle und ihre Probleme bespreche, Musiksamples zu fassen. Daraufhin entwickle ich eine Typologie von Musiksamples, die sich ihrer Flüchtigkeit annimmt, und gehe auf die Rolle des Sample-Clearings genauer ein, mit dessen Hilfe Musiksamples im Musikgeschäft als flüchtige Waren gehandelt werden können. Empirisch analysiere ich aber Onlinemärkte, die den Vertrieb von Samples online organisieren. Mich interessiert, wie diese Märkte mit der flüchtigen Ware Musiksample umgehen und inwiefern das künstlerischen Praktiken entspricht. Dabei zeigt sich, dass Onlineangebote Samples fixieren, um sie als Ware handhabbar zu machen, mit Tracklib aber eine Anbieterin gezielt Praktiken der Samplingkultur integriert, um Samples in gewisser Weise flüchtig zu halten.

Mit Bezug auf die Richtlinie bleibt offen, inwiefern ein Markt harmonisiert werden kann, dessen Waren nur zum Teil verstanden werden. Zwar setzt auch die Richtlinie, basierend auf nationalstaatlichen Rechten, auf temporäre Monopolisierung kultureller Produkte wie Musikaufnahmen. Bis wohin die Monopolbefugnisse reichen, ist allerdings umstritten. Dieser Unsicherheit spüre ich nach und charakterisiere Lizenzierungen, einer der zentralen Bausteine der Reform, als Tipping Point im Urheberrecht, die den flüchtigen Warencharakter von Musiksamples als Stellschraube so-

8 Klammt, Samples 2010, 1, 5.

9 Katz, Capturing Sound, 149.

10 Schumacher Media, Culture \& Society 1995, 253, 261.

$11 \mathrm{Katz}$, Capturing Sound, 148. 
wohl in Richtung einer erweiterten Ermöglichung oder aber vergrößerten Einschränkung verändern kann.

\section{Märkte und Waren}

Um die Organisation von Märkten für die flüchtige Ware Musiksample zu untersuchen, sind Perspektiven der Wirtschaftssoziologie hilfreich. Märkte dienen für diese dazu, Rechte auszutauschen, Angebote zu evaluieren und mit Preisen zu versehen ${ }^{12}$. Das Interesse der Käuferinnen ${ }^{13}$ und Verkäuferinnen $\mathrm{zu}$ handeln ist entscheidend für die Konstitution von Märkten ${ }^{14}$, wobei diese Akteurinnen immer die Wahl haben „to trade, sell or buy at the price they are offered, but they do not have to" ${ }^{15}$. Während die klassische Wirtschaftswissenschaft von einem Markt ausgeht, spricht sich die soziologische Analyse für ein Nebeneinander von Märkten aus. Ökonomischer Wert wird auf Märkten in Preisen angegeben, durch die Produkte miteinander vergleichbar werden. Märkte sind geordnet, wenn sie es erlauben, Vorhersagen über einen Preis zu treffen. ${ }^{16}$ Um Waren einen Preis zuzuordnen, müssen diese kalkulierbar sein. Die Effektivität eines Marktes basiert darauf, inwiefern er in der Lage ist, Kalkulationen im weiten Sinn möglich zu machen:

Calculation starts by establishing distinctions between things or states of the world, and by imagining and estimating courses of action associated with those things or with those states as well as their consequences. ${ }^{17}$

Um Waren kalkulierbar zu machen, müssen diese in eine Objektform gebracht werden und von anderen Objekten abgelöst wahrnehmbar sein. Dazu werden sie gegenüber anderen Objekten vereinzelt, um sie vergleichen und unterscheiden zu können. Prozesse wie „classification, clustering and sorting "18 bringen Waren in Zusammenhang mit anderen Waren und ermöglichen Vergleiche sowie eine Zuordnung eines Preises.

12 Aspers, Markets, 4.

13 Aus Gründen der Lesbarkeit verwende ich durchgehend die weibliche Form.

14 Swedberg Theory and Society 2003, 1, 6.

15 Aspers MPIfG Working Paper 2009, 1, 7.

16 ebd.

17 Callon/Muniesa Organization Studies 2005, 1229, 1231.

18 A.a.O., 1235. 
Auf Märkten der Kreativindustrien sträuben sich Waren, auf diese Weise kalkulierbar zu sein. Waren wie Musikstücke können als inkommensurabel, also prinzipiell unvergleichbar angesehen werden. ${ }^{19}$ Diese Unvergleichbarkeit rührt aus ihrer essentiellen Einzigartigkeit, weshalb wirtschafts- aber auch kultursoziologische Forschung von Singularitäten spricht $^{20}$. Mit Singularität geht Qualitätsunsicherheit einher, die sich in Unsicherheit über Preise wiederspiegelt. Sogenannte ,judgement devices“21 dienen dazu, Unsicherheiten zu reduzieren. Das sind Technologien wie Rankings oder Kritikerinnen, die gewisse Ordnungen in prinzipiell Unvergleichbares bringen. Mit Hilfe dieser Bewertungspraktiken werden Singularitäten auf Märkten gehandelt. Unvergleichbarkeit und Preisunsicherheit zeichnen auch flüchtige Musiksamples aus, weshalb diese durchaus als Singularitäten angesehen werden können. Bei Samples kommt allerdings hinzu, dass sie häufig erst in kreativen Akten der Auswahl aus vorherbestehendem Musikmaterial hergestellt werden, was sie von typischen Singularitäten unterscheidet. Für Musiksamples können zudem Clearing Agents als judgment devices angesehen werden, auf die ich im nächsten Kapitel zu sprechen komme.

Polanyis Konzept fiktiver Waren trägt zur Analyse einen weiteren Aspekt bei, da dieses darauf aufmerksam macht, dass Grenzbereiche dessen existieren, was wir als Waren auffassen. ${ }^{22}$ Bei fiktiven Waren handelt es sich um Güter wie Land, Arbeit oder Geld, die nie als Waren für Märkte produziert wurden und doch auf solchen zu finden sind. Ähnliches lässt sich über Musiksamples sagen, die als Teil eines singulären Musikstücks und nicht als flüchtiges Sample produziert worden sind.

Märkte dienen zusammengefasst dazu, Waren kalkulier- und damit vergleichbar zu machen. Bestimmte Waren entziehen sich einer Kalkulierbarkeit und werden über Bewertungstechnologien und nur unvollständig vergleichbar gemacht. Andere Waren machen den Eindruck, gar keine Waren zu sein, und werden dennoch auf Märkten gehandelt, manche Objekte können über Zeit einen Warencharakter entwickeln, den sie ursprünglich nicht hatten. Musiksamples können als Kombination aus fiktiven Waren und Singularitäten verstanden werden. Dabei kommen allerdings in der Analyse sowohl die Fragmentierung, und die aus ihr erwachsende Unsicherheit, als auch der ästhetische Moment der Herstellung von Musiksam-

19 Karpik, Valuing the Unique, 3.

20 Reckwitz, Die Gesellschaft der Singularitäten, 12, Karpik, Valuing the Unique, 3.

21 Karpik, Valuing the Unique, 44.

22 Polanyi, The Great Transformation, 71. 
ples zu kurz, weshalb ich vorziehe, mit Bezug auf Samples von flüchtigen Waren zu sprechen.

\section{Musiksamples und Praktiken des Samplings}

Sampling, und damit die Nutzung von Samples in Musikproduktionen, hat sich als eine wesentliche Kreativpraktik kontemporärer Musik etabliert. ${ }^{23}$ Musiksamples verstehe ich als Teile zuvor fixierter Musik, die in neue Musikstücke integriert werden. Samples können durch Auswahl und Kuratierung einzigartiger Teile vorherbestehender Musik hergestellt werden. Sampling-Künstlerinnen nutzen dafür u.a. das crate digging als kreative Praktik und wühlen sich nicht nur bildlich gesprochen durch Massen an vorbestehender Musik. ${ }^{24}$ Mittels Praktiken des cutting und mixing ${ }^{25}$ und ebenso digitaler Transformationstechniken, kreieren sie aus Samples neue Musiken.

Im Gegensatz zu den ästhetischen Praktiken sind die rechtlichen Rahmenbedingungen für diese Nutzung vorherbestehender Musik seit Jahrzehnten unsicher. Rechtliche Unsicherheit wird als Hindernis für kreative Prozesse $^{26}$ oder als Möglichkeitsraum für kreative Umgehungsstrategien beschrieben ${ }^{27}$. Unklar ist, wie man an Samples gelangen kann, ohne Rechte Dritter zu verletzen. Akte der rechtssicheren Aneignung in Form von Lizenzierungen sind unübersichtlich. ${ }^{28} \mathrm{Um}$ an eine Lizenz zu kommen, müssen die Rechte aller an der Komposition Beteiligten sowie der Rechteinhaberinnen der Tonträgerherstellungsrechte geklärt werden, da ein Sample sowohl die konkrete Aufnahme nutzt als auch potentiell die Komposition. Zahlreiche Gerichtsurteile haben sich - meist in restriktiver Weise - mit Sampling befasst, ohne im Ergebnis Recht und Musikpraktik in Einklang zu bringen. Zwar liegt für den Sampling-Fall Metall auf Metall zum ersten Mal ein Urteil des Europäischen Gerichtshofs (EuGH) vor. Ob das eingeführte Kriterium der „beim Hören nicht wiedererkennbare[n] Form“ ${ }^{29}$ tatsächlich zu mehr Rechtssicherheit oder einer Anerkennung ästhetischer Praktiken führt, bleibt abzuwarten.

23 Fischer, Sampling, 13.

24 Abmed/Benford/Crabtree Digging in the crates 2012, 1805, 1805.

25 Hebdige, Cutn'Mix, 10.

26 Schumacher Media, Culture \& Society 1995, 253, 266.

27 Fischer, Sampling, 297.

28 McLeod/DiCola, Creative license, 75.

29 EuGH, 29.07.2019 - C-476/17, 56 - Metall auf Metall. 


\subsection{Typologien von Musiksamples}

Um Samples zu differenzieren und in welcher Form diese auf Onlinemärkten angeboten werden, sind Typologien hilfreich. Sewell ${ }^{30}$ kategorisiert Musiksamples hinsichtlich ihrer instrumentellen Nutzung und zeigt, wie sich Sampling im Licht restriktiver Rechtsprechung über Zeit verändert hat. Sie unterscheidet unter anderem zwischen strukturellen Samples, die als Patterns einen Song unterlegen, und Surface-Samples, die der Betonung oder Ausschmückung dienen. Ihre Analyse zeigt, dass Surface-Samples aufgrund der rechtlichen Situation seltener genutzt werden. Fischer hingegen analysiert, inwiefern ein Musiksample in einem neuen Musikstück erkennbar bleibt und bezeichnet die kontinuierliche Abstufung der Erkennbarkeit als „50 shades of referentiality“31. Auch das Urteil des EuGH unterstreicht die allgemeine Bedeutung dieser Dimension für Musiksamples. Andere Autorinnen schlagen ein ästhetisches, aber auch ethisches Kontinuum bei der Betrachtung von Samples vor ${ }^{32}$. Ich möchte ergänzen, Musiksamples dahingehend zu befragen, auf welche Weise sie hergestellt und zu Samples gemacht worden sind und unterscheide vier Szenarien, die ich hinsichtlich ihrer Referentialität und Flüchtigkeit anordne. Flüchtigkeit bezieht sich wiederum auf die Fragmentierung sowie die Performativität der Herstellung eines Samples, Referentialität hingegen darauf, ob ein Musiksample mit Bezug zu einer vorherbestehenden Referenz hergestellt worden ist oder unabhängig von einer solchen.

\section{Referentialität}

$$
\text { Flüchtigkeit niedrig hoch }
$$

$\begin{array}{ccc}\text { niedrig } & \text { vorgefertigtes Sample } & \text { eingespieltes Sample } \\ \text { hoch } & \text { replayed Sample } & \text { kuratiertes Sample }\end{array}$

\section{Abbildung 1: Typen von Samples}

Zumeist ist von kuratierten Samples die Rede, wenn es um rechtliche Unsicherheiten geht. Ebenso finden kreative Praktiken des Samplings häufig im Zusammenhang kuratierter Samples statt. Kuratierte Samples entstehen, wenn eine Musikerin sie aus vorherbestehendem Material hervorbringt. Sie sind deshalb sowohl besonders flüchtig und weisen ebenso

30 Sewell Journal of Popular Music Studies 2014, 295, 304.

31 Fischer, Sampling, 25.

32 Behr/Negus/Street Journal of Cultural Research 2017, 223, 227. 
einen hohen Grad an Referentialität auf. Zwei Musikerinnen können aus demselben Musikstück zwei (und grundsätzlich unzählige mehr) unterschiedliche Musiksamples kreieren. Kuratierte Samples müssen für gewöhnlich lizenziert und rechtlich geklärt werden.

Es ist auch möglich, vorgefertigte Samples zu erwerben. Sample Packs oder Sample Libraries bestehen aus tausenden kurzen Samples, Loops oder One-Shots, die in Musikstücken genutzt werden dürfen. Diese Samples sind in großer Zahl auf digitalen Märkten anzutreffen und mit Nutzungslizenzen ausgestattet. Vorgefertigte Samples weisen nur in Ausnahmefällen Referenzialität auf und sind kaum als flüchtig anzusehen, da sie kein Fragment darstellen. Diese beiden Typen sind für Sample-Märkte entscheidend und ich werde mich im Folgenden hauptsächlich auf sie beziehen.

Darüber hinaus kommen selbst eingespielte Samples häufig zum Einsatz. So können Multiinstrumentalistinnen mit ihrer Hilfe komplexe Musiken alleine produzieren. Eingespielte Samples können schließlich in Form von replayed Samples dazu verwendet werden, Tonträgerrechte zu umgehen. Das passiert, wenn ein Sample nicht geklärt, aber nachgespielt werden kann. Bei diesen ist die Referentialität wiederum hoch, sollen sie ja klingen wie das Original, sie sind aber in geringerem Maße flüchtig, da für ihre Produktion schon ein spezifischer Ausschnitt vorgegeben sein muss, sie also nicht mehr performativ entstehen.

\subsection{Sample-Clearing und rechtliche Unsicherheit}

Um das Problemfeld der Märkte für Musiksamples als flüchtige Waren abzustecken und damit auf ihre Onlinemärkte hinzuführen, stütze ich mich auf Aussagen aus Gerichtsurteilen und Interviews mit Clearing-Agents. Diese sind Branchenkennerinnen, die aufgrund ihrer Spezialisierung Einschätzungen zu Marktpraktiken beisteuern und aufzeigen, wie mit den flüchtigen Waren im Musikgeschäft umgegangen wird. In ihren Ausführungen nimmt eine Expertin für das Klären von Musiksamples fast ausschließlich Bezug auf kuratierte Samples und fasst die Situation am Markt zusammen:

I've been doing it for 25 years (...). Back then we used to be able to do it for a lot less. When Common did an album, like his first album, he would have four or five samples in a song and it would be a challenge, but we would be able to make it happen. You just can't do it anymore because everyone's so greedy and they [the publishers] want a minimum of 10 or 15 percent. So, it makes it very difficult to be able to 
take a song and sprinkle it in, which is what producers like DJ Premier used to do. ${ }^{33}$

Zwar meint die Agentin, Sampling solle keine Praktik für finanzkräftige Künstlerinnen sein, gibt aber an, dass für Albumproduktionen mit Samples sechsstellige Summen für deren Klärung nötig sind. In seltenen Fällen werden einzelne Samples für derartige Beträge gehandelt. Allgemein nimmt die Agentin einen Anstieg der Preise für Samples wahr. Sie erklärt das damit, dass große Teile des Musikrepertoires sich durch das Aufkaufen von Katalogen in der Hand weniger Major Labels befinden. Früher standen viele kleine Rechteinhaberinnen sampelnden Künstlerinnen gegenüber. Das gab auch beim Clearing mehr Verhandlungsspielraum. Neben der Gier von Verlagen und Labels, spielt die freie Entscheidung jeder Rechteinhaberin eine Rolle, Sampling ohne Nennung von Gründen abzulehnen. Die Expertin betont dennoch ihre hohe Klärungsquote, die auf Feldwissen basiert:

I'll give free advice to my clients so they have an idea of if what they want to sample is feasible. I'll say "that copyright holder isn't clearable," (...), it's going to be costly or time consuming. After 25 years, I know who clears and who doesn't, who's quick, who's difficult, all that kind of stuff. 34

Wenn sich ein Sample klären lässt, müssen Musikerinnen ihre fertigen Stücke vorlegen, um eine monetäre Bewertung des Samples zu ermöglichen. Eine nachträgliche Veränderung mit geklärten Samples ist nur durch eine erneute Klärung möglich. Zwar soll die Bekanntheit einer Musikerin nicht auf den Preis eines Samples Einfluss nehmen, wenig bekannte Musikerinnen verfügen aber über eine schlechtere Position, um an Samples zu gelangen. Die Agentin schlägt Musikerinnen mit geringen finanziellen Mitteln vor, nachgespielte Samples zu produzieren, um keine Aufnahmerechte klären zu müssen und den Gesamtpreis zu reduzieren. Diese Annahmen über den Markt für Musiksamples stehen in Diskrepanz zu Äußerungen des berühmten „Get a license or do not sample“-Urteils:

33 Garrison, This Woman Clears the Samples on Your Favorite Albums, Here's How, https://djbooth.net/features/2015-05-24-how-sample-clearance-works, (zuletzt abgerufen am 18.04.2020).

34 ebd. 
The market will control the license price and keep it within bounds. The sound recording copyright holder cannot exact a license fee greater than what it would cost the person seeking the license to just duplicate the sample in the course of making the new recording. ${ }^{35}$

Für die Annahme, dass Lizenzierungen nicht teurer sein würden als das Nachspielen einer Aufnahme, gibt es keine Anzeichen. Vielmehr ist die Singularität eines Originals bestimmend für seinen Wert, sowohl für Sampling-Künstlerinnen als auch für Rechteinhaberinnen. Während die einen einzigartige Samples verwenden wollen, sehen die anderen in ihren alten Aufnahmen Schätze verborgen, deren Wert auch auf der Rarheit von Pressungen basieren kann. Auch in anderen Gerichtsurteilen zeigt sich, dass rechtliche Akteurinnen mit vereinfachten Annahmen über den Markt für Samples urteilen:

Dagegen ist die Freiheit der Künstler meines Erachtens weit weniger umfangreich, was den Erwerb der für ihr Schaffen benötigten Mittel betrifft. Jeder Künstler muss sich mit den Bedingungen des Lebens in der Gesellschaft und des Marktes, auf dem er tätig ist, abfinden. Die Freiheit der Kunst befreit die Künstler nicht von den Zwängen des Alltags. Wäre es vorstellbar, dass ein Künstler sich auf seine schöpferische Freiheit berufen könnte, um seine Farben und Pinsel nicht bezahlen zu müssen. ${ }^{36}$

Eine Malerin muss Pinsel und Farbe kaufen, um malen zu können. Nach dieser Analogie muss eine Musikerin Samples kaufen, um Musik zu machen. Dabei wird ignoriert, dass Pinsel und Farben zu festen Preisen erworben werden können, die sich kaum zwischen Anbietenden unterscheiden. Vor allem für kuratierte Samples gibt es aber keine Geschäfte, sie existieren nicht einmal in Form einer Ware, bevor sie eine Musikerin auswählt und damit herstellt, was sie den fiktiven Waren Polanyis annähert. Erschwerend kommt hinzu, dass es bei einem Sample rechtlich unsicher ist, $\mathrm{ob}$ es sich dabei um ein künstlerisches Werkzeug wie einen Pinsel, ein künstlerisches Material wie Farbe oder doch eigentlich um ein - trotz Fragmentierung - musikalisches Produkt handelt, dessen potentiell urheberrechtlich geschützter Status als Werk unklar ist ${ }^{37}$.

35 Court of Appeals 6th Circuit, 28.04.2004 - 383 F. 3d 390, 399 - Bridgeport Music, Inc. v. Dimension Films.

36 Generalanwalt beim EuGH, 12.12.2018 - C-476/17, 92, ZUM 2019, 237, Rn. 92 Metall auf Metall.

37 Sinnreich, Mashed Up, 171. 
Auf die wirtschaftliche Bedeutung von Samples weisen auch die Rechteinhaberinnen hin. So erwähnen die Klägerinnen des Metall auf MetallFalls, dass der Markt für Samples vormals nicht wichtig erschien, doch heute große Bedeutung hat. ${ }^{38}$ Musikpraktiken verändern sich und mit ihnen die Waren, die auf Musikmärkten gehandelt werden. Aus Musikstücken, die auf Langspielplatten oder im Radio als singuläre, abgrenzbare Waren zirkulierten, sind heute solche geworden, die sich auch als potentielle Reservoirs für kreative Lizenzierungen durch Musiksamples verstehen. Neben den zahlreichen Zweit- und Drittnutzungen wie Synchronisationen oder die Produktion von Cover-Versionen, die das Musikgeschäft schon kennt, entwickelt sich in der Fragmentierung von Musikstücken ein Markt für Musiksamples als flüchtige Waren.

Die Schlaglichter aus dem Feld zeigen, dass Märkte für Musiksamples existieren und sie in gewisser Weise dem entsprechen, was die wirtschaftssoziologische Analyse von Märkten erwartet. Allerdings unterstreichen Eigenheiten des Sample Clearings, wie schwierig Samples für viele Musikschaffende zu handhaben sind. Ihre Flüchtigkeit verhindert eine eindeutige Bewertung, da der konkrete Preis eines Samples erst nach Verwendung ermittelt wird. Ein und dasselbe Sample kann in zwei musikalischen $\mathrm{Zu}$ sammenhängen andere Preise haben. Da Musiksamples erst als Waren existieren, nachdem sie von einer Musikerin als ein Musiksample identifiziert und verwendet worden sind, zeichnen sie sich besonders dadurch aus, dass erschaffende und kaufende Akteurin in eine Person fällt. Niemand auf dem Markt für Musiksamples weiß mit Sicherheit, wie es denn tatsächlich um ein Sample steht. Aber es bestehen Erfahrungswerte, die eine Einschätzung erleichtern, wie die Agentin ausgeführt hat. Dennoch wissen die Besitzenden bzw. potentiell Verkaufenden nicht genau, im Besitz welcher flüchtigen Waren sie sind, denn diese müssten zunächst von den Sampling-Künstlerinnen ausgewählt und genutzt werden. Die Samplenutzerinnen wissen nicht genau, was ein Sample kosten wird oder ob es geklärt werden kann. Das wissen, wenn überhaupt, nur Expertinnen mit langjähriger Felderfahrung. Gewissheit steuern Akteurinnen bei, die sich definitiv gegen jedes Sampleclearing stellen. Unter anderem Prince ist dafür bekannt.

38 BVerfG, 31.05.2016 - 1 BvR 1585/13, Rn. 55 - Metall auf Metall. 


\section{Mapping digitaler Märkte für Musiksamples}

Nachdem ich die Probleme insbesondere kuratierter Samples auf dem allgemeinen Musikmarkt beschrieben habe, analysiere ich im Folgenden Onlinemärkte für Samples. Mit Hilfe eines Mappings untersuche ich, wie diese mit Samples als Ware und ihrer potentiellen Flüchtigkeit umgehen. Im Frühjahr 2019 habe ich 24 digitale Marktplätze ausgemacht, auf denen Samples angeboten werden. Über Blogeinträge habe ich mich über Onlinemärkte informiert. Ergänzend habe ich Schlagwortsuchen mit Suchbegriffen wie „buy music samples“ oder „get music samples“ durchgeführt. Auf den Marktplätzen selbst habe ich Beschreibungen, Lizenzbedingungen, Preislisten bzw. Praktiken der Preisbildungen gesammelt.

Um einen Überblick über die Marktplätze zu erhalten, habe ich aus meinen Daten induktiv vier Typen von Onlinemärkten differenziert, die sich nicht an der oben vorgestellten Typologie von Samples, sondern an ihrer wirtschaftlichen Organisation orientieren. Das hat damit zu tun, dass die überwiegende Mehrheit der Onlinemärkte für Musiksamples ausschließlich vorgefertigte Samples anbietet und die weiteren differenzierten Sampling-Typen eine untergeordnete Rolle spielen. Ich unterscheide zwischen Onlineshops für Samples \& Samplepacks, Abo Onlineshops, Digitale Sharing-Communities und Digitale Audioproduktionsumgebungen. Diese Einteilungen sind nicht immer trennscharf, es kommt zu Hybriden. Die Trennung von kommerziellem und nicht-kommerziellem Angebot ist mitunter schwammig (Freemium-Angebote). Der Onlinemarkt für Samples befindet sich in Bewegung und ein Mapping bietet immer nur eine Momentaufnahme.

Markttypen

Onlineshops für Samples \& Samplepacks Abo Onlineshops

Digitale Sharing-Communities

Digitale Audioproduktionsumgebungen
Beispiele Marktplätze

Primeloops; Tracklib

Sounds; Noiiz

Looperman; Sampleswap

Splice; Looplabs

Abbildung 2: Onlinemärkte und Marktplatzbeispiele ${ }^{39}$

39 Eine Liste aller im Mapping untersuchten Marktplätze findet sich im Appendix. 
Onlineshops für Samples \& Samplepacks verkaufen vorgefertigte Samples bzw. auch Loops, Sounds oder One-Shots. Diese Samples sind in der Regel kurz, selbst eine einzelne Kick-Drum kann ein Sample sein. Viele der Sample-Packs werden quantitativ mit Argumenten des Überflusses und der Masse beworben, indem die Anzahl der inkludierten Samples hervorgehoben wird, und damit Packs vergleich- und in gewisser Weise kalkulierbar gemacht werden. Andere Anbieterinnen setzen auf die qualitative Einzigartigkeit der Samples in ihren Packages, die von bekannten Produzentinnen stammen. Preise variieren zwar, bewegen sich aber in wenigen, einander ähnlichen Preissegmenten. Häufig sind Samplepacks in thematische Schwerpunkte eingebettet, die bspw. Hinweise auf Genres geben, aus denen Samples stammen. Mit dem käuflichen Erwerb geht eine Nutzungslizenz einher, die den Musikerinnen erlaubt, die Samples in eigenen kommerziellen oder nicht-kommerziellen Produktionen zu verwenden, ohne Rechteklärungen vornehmen zu müssen. Verboten ist es, Samples außerhalb neuer Musikstücke weiter zu verkaufen.

Abo Onlineshops unterscheiden sich in der Art, wie die angebotenen Samples erworben werden. Anstelle einmaliger Käufe, schließen die Kundinnen Abonnements mit den Marktplätzen ab. Gewöhnlich gibt es mehrere Abo-Möglichkeiten, die sich durch den Preis und die Anzahl der Samples differenzieren, die pro Monat heruntergeladen werden dürfen.

Auf Digitalen Sharing Communities können Samples gratis unter den Teilnehmenden getauscht werden. Häufig geht das mit einer Creative Commons-Lizenzierung einher, wobei die Produzentinnen der Samples über den CC-Lizenztyp entscheiden. Im Unterschied zu den beiden zuvor behandelten Markttypen können die Nutzerinnen des Marktes auch die Produzentinnen der Samples sein.

In Digitalen Audioproduktionsumgebungen haben Samples den Charakter von "Goodies“, die einen fixierten Abo-Preis rechtfertigen. Diese Umgebungen sind nicht hauptsächlich mit der Verbreitung von Samples befasst, sondern stellen sich als digitale Kollaborationsplattformen für Musikschaffende dar. Auf diesen Plattformen dürfen Nutzerinnen Samples hochladen, damit sich über Samples Kollaborationen entwickeln oder andere Musikerinnen diese in ihren Produktionen nutzen. Daneben wird mit einzigartigen Samples geworben, die im Abo enthalten und von professionellen Produzentinnen geschaffen worden sind.

Während die kreative Praktik des kuratierten Samples im Auswählen und Herstellen der Samples selbst zu suchen ist und das Zusammensetzen unterschiedlicher Fundstücke einen kreativen Mix ergibt, verändert sich der Diskurs rund um kreatives Sampling auf Onlinemärkten. Kreativität liegt in diesen Märkten auf der Transformation von Samples. Die Anbiete- 
rinnen verweisen darauf, dass sie mit ihren vorgefertigten Samples und Sounds Musikschaffende inspirieren wollen und die Aufforderung zur Transformativität dringt bis in die Lizenzbedingungen vor:

(...) you use the Sound Product(s) only within your own newly-created sound recording(s) in a manner that renders the Sound Product substantially dissimilar to the original sound of the Sound Product in each case. (Noiiz_Terms and Conditions, Pos. 21)

Diese Aufforderung erinnert frappierend an die Formulierung des EuGHs zu rechtskonformem Sampling, der Nicht-Wiedererkennbarkeit. Dieses zeichnet sich offenbar durch eine niedrige Referentialität aus. Das Sample hat fast ausschließlich eine Material-Konnotation, muss in breitem Umfang transformiert werden, um genutzt werden zu dürfen und gleichsam zum eigenen Werk zu werden.

Eine Ausnahme zu diesen Onlinemärkten stellt Tracklib dar. Tracklib ist ein Onlineshop für Samples, der sich an Praktiken kuratierter Samples orientiert und auf vorgefertigte Samples verzichtet. Denn auf Tracklib können aus vollständigen Musikstücken in Form eines digitalen crate diggings Musiksamples kreiert und im Anschluss daran lizenziert werden. Eine kategoriale Einteilung in A, B und C der Musikstücke zeigt an, wieviel die Lizenz für ein Sample aus dem jeweiligen Musikstück kostet. Kategorie A kostet $1500 \$$, B $500 \$$ und C 50\$. Nach eigenen Angaben befinden sich $99 \%$ aller Musikstücke in Kategorie C. Darüber hinaus wird ein prozentualer Anteil an den Revenues eines Songs fällig, der nach der Länge eines Samples im Original variiert. Ein Lizenzierungsschema hilft, den Überblick zu behalten. Die Kategorien beziehen sich auf die originalen Musikstücke, aus denen die Samples kreiert werden können. Wiederum unterscheidet sich der hier prozentuale Preis zwischen den Kategorien deutlich. Die Sekundenangabe bezieht sich auf das Originalstück, Loops können aus Samples gebildet und müssen nicht extra verrechnet werden. 


\begin{tabular}{|lccc|}
\hline SAMPLE LENGtH & $\leq 2$ SEC & $\leq 15$ SEC & $\leq 60$ SEC \\
CATEGORY C & $2 \%$ & $10 \%$ & $20 \%$ \\
CATEGORY B & $5 \%$ & $15 \%$ & $25 \%$ \\
CATEGORY A & $10 \%$ & $25 \%$ & $35 \%$ \\
\hline
\end{tabular}

Abbildung 3: Tracklib Lizenzierungsschema ${ }^{40}$

Musikerinnen können sich über diese Tabelle ausrechnen, wie viele Samples sie verwenden wollen. So könnten in Kategorie C 50 kurze Samples in einem Musikstück integriert werden, bevor 100 \% der Rechte eines Songs abzutreten wären. Dazu kommen $2500 \$$ an Lizenzgebühren. Aus einem Zwei-Sekunden-Sample kann aber der Loop für ein ganzes Lied kreiert werden, es würden weiterhin alleine $2 \%$ der Revenues anfallen. Songs der Kategorie A ausschweifend zu nutzen ist nicht im selben Ausmaß möglich. Hier sind die Rechte an einem neuen Song nach 10 Samples an andere Rechteinhabende aufgeteilt. Auch die Lizenzgebühren von $15.000 \$$ müssten aufgebracht werden.

Tracklib überführt das kuratierte Sample unter vereinfachten und standardisierten, sowie vor allem kalkulierbaren Bedingungen in digitale Märkte. Mit wenigen, einfachen Kalkulationen kann eine Musikerin berechnen, ob sie sich die Nutzung von Samples finanziell leisten kann bzw. einen bestimmten Anteil ihres möglichen eigenen Verdienstes am Song abtreten möchte. Die Anzahl und Länge der Samples sowie eine nicht wei-

40 Anonym, Licensing, www.tracklib.com/licensing/, (zuletzt abgerufen am 27.06.2020).

Als eine empirische Randnotiz: während bei der Erstellung der ursprünglichen Version des Artikels die Lizenzen für Kategorie A Songs bei $2500 \$$ und die prozentuale Beteiligung bei Samples zwischen 15 und 60 Sekunden 20/30/50 \% je nach Kategorie lag, haben sich offensichtlich der Preis der Kategorie A, sowie die Revenue Anteile der Kategorie A und B in den letzten Monaten zum Teil deutlich reduziert. 
ter explizierte qualitative kategoriale Einteilung entscheidet über den Preis. Die additive Kumulation von Revenue-Anteilen führt wiederum dazu, der Flüchtigkeit von Sampling fixierte Grenzen aufzuerlegen. Während die Onlineshops ihren vorgefertigten Samples eine Materialfunktion zuordnen, bewertet Tracklib Samples aus einer Produktsicht. Beides kann zu einer Entflüchtigung von Samplingpraktiken führen.

\section{Die Fixierung flüchtiger Waren}

In meinem Beitrag analysiere ich Samples als flüchtige Waren. Ich beschreibe, welche Probleme auf Märkten damit einhergehen und welche Onlinemärkte sich um den Vertrieb von Samples gebildet haben. Vor dem Hintergrund der Richtlinienreform des digitalen Binnenmarktes in der EU untersuche ich, wie Onlinemärkte mit der potentiellen Flüchtigkeit von Samples umgehen. Musiksamples habe ich dafür hinsichtlich ihrer Herstellungsart differenziert und auf die Unterscheidung zwischen vorgefertigten und kuratierten Samples. Vorgefertigte Samples verfügen über geringe Flüchtigkeit und geringe Referentialität. Kuratierte Samples zeichnet hingegen Flüchtigkeit und Referentialität in hohem Maß aus.

Vordergründig zeigt sich auf den Onlinemärkten für Musiksamples ein vielfältiges Angebot. Es gibt insbesondere aus einer wirtschaftlich-organisationalen Sicht unterschiedliche Möglichkeiten online an Samples zu kommen. Um die flüchtigen Waren allerdings handhaben zu können, kommt es auf Onlinemärkten fast ausschließlich zur Fixierung der Samples in Form von vorgefertigten Samples. Einzelne Angebote bemühen sich zwar darum, den Kreativpraktiken kuratierter Samples zu entsprechen. So simuliert die Plattform Tracklib ein online crate digging, reduziert dabei aber die maximale Samplingkapazität eines Songs und verknüpft die ästhetische Nutzung von Samples mit wirtschaftlichen Grenzziehungen. Dadurch fixiert dieses flüchtigere Angebot auf einer formalen, wirtschaftlichen Ebene die Flüchtigkeit der Sampling-Nutzung. Mit den Fixierungen einher gehen allgemein standardisierte Lizenzierungen bzw. kalkulierbare Bedingungen, die rechtliche Unsicherheiten auf Onlinemärkten für Samples reduzieren. Damit unterscheiden sich Onlinemärkte von dem Markt für Musiksamples im Musikgeschäft, die auf individuelle Rechteklärung setzen und in denen rechtliche Unsicherheit allgegenwärtig ist.

Das Konzept der flüchtigen Waren kann als durchaus tauglich angesehen werden, die Ware Musiksample genauer zu analysieren. Es zeigt, wie und dass der Herstellungsprozess eines Samples, die Auswahl und Kuratierung musikalischer Fragmente, eine Rolle spielt und verknüpft das mit dem 
Umgang mit Samples als Waren auf verschiedenen Märkten. In der Flüchtigkeit von Samples liegt eine wesentliche performative und ästhetische Dimension, die aber zu rechtlichen und wirtschaftlichen Unsicherheiten führt. Allerdings haben sich auch Grenzen der Nützlichkeit des Konzepts gezeigt. So müsste noch genauer untersucht werden, wie das Musiksample als flüchtige Ware mit anderen, etablierten Zweit- und Drittnutzungen im Musikgeschäft in Verbindung steht. Das habe ich in diesem Beitrag nicht ausreichend geklärt.

Die digitalen Märkte bringen zwar eine Vielzahl an Möglichkeiten hervor, an potentiell flüchtige Waren wie Samples zu kommen, tatsächlich flüchtig werden die Waren bisher aber noch nicht. Die potentielle Performativität digitaler Inhalte und ihre Möglichkeiten zu Formen von Remix sind mit Musiksamples sicherlich nicht ausgeschöpft. Während es möglich ist, dass in Zukunft vermehrt visuelle Samples für Remix bedeutsam werden, denke ich ebenso an die Performativität von Programmiercode. Im Musikgeschäft tauchen vermehrt Hologramme auf, die für Unterhaltung sorgen. ${ }^{41}$ Diese synästhetischen Kulturprodukte machen flüchtige Waren denkbar, die zum Material äußerst spannender Kulturprodukte werden könnten. Auch hier werden Lizenzierungen ermöglichend oder doch einschränkend wirken.

41 Anonym, Productions, https://basehologram.com/productions (zuletzt abgerufen am 27.06.2020). 


\section{Literatur}

Abmed, Abmed/Benford, Steve/Crabtree, Andy: Digging in the crates: an ethnographic study of DJS'work, Proceedings of the SIGCHI conference on human factors in computing systems (2012), S. 1805 ff., doi:10.1145/2207676.2208314.

Anonym, Licensing, www.tracklib.com/licensing/, (zuletzt abgerufen am 27.06.2020).

Anonym, Productions, https://basehologram.com/productions (zuletzt abgerufen am 27.06.2020).

Aspers, Patrik: How are markets made?, MPIfG Working Paper 2 (2009), S. 5 ff.

Aspers, Patrik, Markets, Cambridge 2011.

Bauman, Zygmunt, Liquid modernity, Chichester 2013.

Behr, Adam/Negus, Keith/Street, John: The sampling continuum: musical aesthetics and ethics in the age of digital production, Journal of Cultural Research 21 (2017), S. 223 ff., doi: 10.1080/14797585.2017.1338277.

Boltanski, Luc/Esquerre, Arnaud, Bereicherung: Eine Kritik Der Ware. Frankfurt a. M. 2019.

Callon, Michel/Muniesa, Fabian: Peripheral vision: Economic markets as calculative collective devices, Organization Studies 26 (2005), S. 1229 ff., doi: 10.1177/01708 40605056393.

Caves, Richard E., Creative industries: Contracts between art and commerce, Cambridge 2000.

Fischer, Georg, Sampling in der Musikproduktion. Das Spannungsfeld zwischen Urheberrecht und Kreativität, Marburg 2020.

Fischer-Lichte, Erika, Ästhetik des Performativen. Frankfurt a. M. 2012.

Goodman, Ellen P.: Media policy out of the box: Content abundance, attention scarcity, and the failures of digital markets, Berkeley Technology Law Journal 19 (2004), S. 1389 ff., doi: dx.doi.org/10.2139/ssrn.590425.

Garrison, Lucas, This Woman Clears the Samples on Your Favorite Albums, Here's How, https://djbooth.net/features/2015-05-24-how-sample-clearance-works, (zuletzt abgerufen am 18.04.2020).

Hebdige, Dick, Cutn'Mix: Culture, Identity and Caribbean Music, London 2003 [1987].

Hondros, Konstantin, Justifying music practices under regulatory uncertainty over time: Analyzing the 'never-ending' court case "Metall auf Metall", Organized Creativity Discussion Paper 20 no. 1 (2020): S. 1 ff., https://www.wiwiss.fu-berlin .de/forschung/organized-creativity/3_publications/Disc-Paper/Hondros_2020_Ju stifying-music-practices-under-regulatory-uncertainty_final.pdf.

Karpik, Lucien, Valuing the Unique. The Economics of Singularities, Princeton 2011.

Katz, Mark, Capturing sound: how technology has changed music, Berkeley 2010. 
Klammt, Sascha: Das Sample. Eine einzigartige Momentaufnahme als Basis für eine neue Komposition, Samples. Online-Publikation der Gesellschaft für Popularmusikforschung 9 (2010), S. $1 \mathrm{ff}$.

Knobel, Michele/Lankshear, Colin: Remix: The art and craft of endless hybridization, Journal of adolescent \& adult literacy, 52 (2008), S. 22 ff., doi: 10.1598/JAAL.52. 1.3 .

McLeod, Kembrew/DiCola, Peter, Creative license: The law and culture of digital sampling. Durham and London 2011.

Polanyi, Karl, The Great Transformation. The Political and Economic Origins of Our Time, Boston 2001 [1944].

Reckwitz, Andreas, Die Gesellschaft der Singularitäten. Zum Strukturwandel der Moderne, Frankfurt a. M. 2018.

Schumacher, Thomas G: 'This is a sampling sport': ${ }^{1}$ digital sampling, rap music and the law in cultural production, Media, Culture \& Society 17 (1995), S. $253 \mathrm{ff}$., doi: 10.1177/016344395017002006.

Sewell, Amanda: How Copyright Affected the Musical Style and Critical Reception of Sample-Based Hip-Hop, Journal of Popular Music Studies 26 (2014), S. 295 ff., doi: 10.1111/jpms.12078.

Sinnreich, Aram, Mashed up: Music, technology, and the rise of configurable culture. Amherst 2010.

Swedberg, Richard: The case for an economic sociology of law, Theory and Society 32 (2003), S. $1 \mathrm{ff}$.

Zanetti, Sandro/Heine, Stefanie, Transaktualität. Ästhetische Dauerhaftigkeit und Flüchtigkeit, München 2017. 
Appendix

Alphabetische Liste des Mappings von Onlinemärkten für Musiksamples; Kürzel: Onlineshop für Samples \& Samplepacks (OS); Abo Onlineshop (AO); Digitale Sharing-Community (DC); Digitale Audioproduktionsumgebung (DA):

\begin{tabular}{ll} 
Marktplatz & Markt \\
\hline www.audiotool.com & DA \\
www.bigfishaudio.com & OS \\
www.freesound.org & DC \\
www.freetousesounds.com & OS \\
www.ghosthack.de & OS \\
www.landr.com & DA \\
www.looplabs.com & DA \\
www.looperman.com & DC \\
www.loopmasters.com & OS \\
www.magix.com & DA \\
www.noiiz.com & AO \\
www.primeloops.com & OS \\
www.producerloops.com & OS \\
www.riemannkollektion.com & OS \\
www.sampleism.com & OS \\
www.sampleswap.org & DC \\
www.samplephonics.com & OS \\
www.sampulator.com & DA \\
www.soundation.com & DA \\
www.sounds.com & AO \\
www.soulsurplus.com & OS \\
www.splice.com & DA \\
www.tracklib.com & OS \\
www.vsl.co.at (Vienna Symphonic Library) & OS \\
& \\
w & \\
w &
\end{tabular}

